\title{
Multistage Security-Constrained HVAC/HVDC Transmission Expansion Planning With a Reduced Search Space
}

\author{
Andres Hernando Dominguez, Leonardo H. Macedo ${ }^{(0)}$, Student Member, IEEE, Antonio Hernando Escobar, \\ and Rubén Romero ${ }^{\mathbb{D}}$, Senior Member, IEEE
}

\begin{abstract}
This paper proposes a new method to solve the multistage security-constrained transmission expansion planning problem, incorporating lines based on high-voltage alternating current (HVAC) and high-voltage direct current (HVDC) alternatives. A novel mixed-integer linear programming model, which incorporates transmission losses using a piecewise linearization, is presented. An efficient method to reduce the search space of the problem is developed to help in the solution process. Garver's 6-bus system and a modified Southern Brazilian system are used to show the precision and efficiency of the proposed approach. The tests are performed for cases with and without HVDC links and transmission losses. The results indicate that better expansion plans can be found by considering HVDC proposals in the expansion process. The promising trend of using HVDC lines in future networks to improve the reliability in the system is demonstrated.
\end{abstract}

Index Terms-HVDC lines, mixed-integer linear programming, multistage transmission expansion planning, power losses, security constraints.

\section{NOMENCLATURE}

The notation used throughout this paper is reproduced below for quick reference.

Indices:

$c \quad$ Index for a contingency scenario

$i, j, k$ Indices for buses

$k i, i j \quad$ Indices for corridors

$l \quad$ The $l$ th block of the piecewise linearization

$t \quad$ Index for a decision stage

$y \quad$ Index for a candidate line option

Sets:

$\Omega_{a c}, \Omega_{d c}$ Set of HVAC/HVDC transmission corridors

$\Omega_{B} \quad$ Set of buses

Manuscript received August 26, 2016; revised December 23, 2016; accepted February 10, 2017. Date of publication February 14, 2017; date of current version October 18, 2017. This work was supported in part by the Colombian agency COLCIENCIAS under Grant 567-2012 and in part by the Brazilian agencies CNPQ, CAPES, and the São Paulo Research Foundation under Grant 2014/23741-9. Paper no. TPWRS-01304-2016.

A. H. Dominguez and A. H. Escobar are with the Department of Electrical Engineering, Technological University of Pereira, Pereira 660003, Colombia (e-mail: andreshdominguez@gmail.com; aescobar@utp.edu.co).

L. H. Macedo and R. Romero are with the Department of Electrical Engineering, São Paulo State University, Ilha Solteira 15385-000, Brazil (e-mail: leohfmp@gmail.com; ruben@dee.feis.unesp.br).

Color versions of one or more of the figures in this paper are available online at http://ieeexplore.ieee.org.

Digital Object Identifier 10.1109/TPWRS.2017.2669323
C

$C^{0}$

$C^{a c}, C^{d c}$

$C I, C I I$

$T$

$Y^{a c}, Y^{d c}$

Set of contingency scenarios $\left(C^{0} \cup C^{a c} \cup C^{d c}\right)$

Operation scenario without contingency

Set of HVAC/HVDC contingency scenarios

Contingency scenarios in corridors with at least one existing line and in corridors with no line

Set of investment stages

Functions:

$f(z, \bar{z}, L) \quad$ Approximation of the square value of $z$

$I C_{t}, C L_{t}$ Investment and losses costs at stage $t$

$P_{c, t}^{l s} \quad$ Power losses in contingency scenario $c$, stage $t$

\section{Constants:}

$\alpha$

$\delta_{t}^{i}, \delta_{t}^{f}$

$\Delta_{c}^{a c}, \Delta_{c}^{d c}$

$\bar{\theta}$

$\lambda_{t}$

$\sigma$

$C_{i j}^{a c}, C_{i j}^{d c}$

$C^{e}$

$d_{i, t}$

$\bar{g}_{i, t}$

$L$

$L C_{i j}$

$n_{i j}^{0, a c}, n_{i j}^{0, d c}$

$\bar{n}_{i j}^{a c}, \bar{n}_{i j}^{d c}$

$N C_{i j, c}^{a c}, N C_{i j, c}^{d c}$

$\bar{P}_{i j}^{a c}, \bar{P}_{i j}^{d c}$

$r_{i j, c}^{a c}, r_{i j, c}^{d c}$

$r_{i j, y, c}^{a c}, r_{i j, y, c}^{d c}$
Annual discount rate

Initial and final operation years of stage $t$

Annual system operation times in scenario $c$

Maximum voltage phase angle limit

Load factor at stage $t$

Load shedding cost

Cost of an HVAC/HVDC line in corridor $i j$

Energy cost

Active power demand at bus $i$, stage $t$

Maximum limit of active power generation at bus $i$, stage $t$

Number of blocks in the piecewise linearization

Capacity loss of an HVDC line in corridor $i j$

Number of existing HVAC/HVDC lines in corridor $i j$

Maximum number of new HVAC/HVDC lines allowed in corridor $i j$

State of an existing or candidate HVAC/HVDC line in corridor $i j$, scenario $c$

Limit of active power flow in HVAC/HVDC corridor $i j$

Equivalent resistance of HVAC/HVDC existing lines in corridor $i j$, scenario $c$

Equivalent resistance of HVAC/HVDC line $y$ in corridor $i j$ under contingency scenario $c$ 


$\begin{array}{ll}S^{b} & \text { Power base } \\ S l_{z, l} & \text { Slope of the } l \text { th discretization of } z \\ x_{i j} & \text { Reactance of an HVAC line in corridor } i j \\ \bar{z} & \text { Maximum limit of } z\end{array}$

Continuous Variables:

$\Delta_{z, l} \quad$ Value of the $l$ th block associated with the discretization of $z$

$\theta_{i, c, t} \quad$ Voltage phase angle at bus $i$, scenario $c$, stage $t$

$\varphi_{i, c, t}$

$g_{i, c, t}$

$I_{i j}^{a c}$

$P_{i j, c, t}^{0, a c}, P_{i j, c, t}^{0, d c}$

Load shedding at bus $i$, scenario $c$, stage $t$

Active power generation at bus $i$, scenario $c$, stage $t$

Current magnitude at HVAC corridor $i j$

Active power flow in the existing HVAC/HVDC line in corridor $i j$, scenario $c$, stage $t$

$P_{i j, y, c, t}^{a c}, P_{i j, y, c, t}^{d c}$ Active power flow in HVAC/HVDC line $y$ in corridor $i j$, scenario $c$, stage $t$

$Q_{i j}^{a c} \quad$ Reactive power flow arriving at bus $j$

$V_{j} \quad$ Voltage magnitude at bus $j$

$z \quad$ Argument of function $f$ that represents a power flow variable

$z^{+}, z^{-} \quad$ Auxiliary variables to calculate $|z|$

Binary Variables:

$w_{i j, y, t}^{a c}, w_{i j, y, t}^{d c}$ Represent the construction of the HVAC/HVDC line $y$ in corridor $i j$ at stage $t$

\section{INTRODUCTION}

$\mathbf{T}$ HE objective in the transmission network expansion planning (TNEP) problem is to determine the least-cost investment in the devices to be added to the transmission network in order to guarantee a proper system operation in the future. Common types of investments are transmission lines and transformers [1]. The problem can be classified as static or multistage [2]. The static approach performs a single investment at the beginning of the planning horizon and determines where and how many devices should be installed; with such an approach, only one planning horizon is analyzed. In the multistage approach, the most appropriate times must be defined to carry out investments in new equipment; this approach must consider the continuous growth of generation and demand, so that the investment and changes in generation and demand are assimilated by the system in an optimized way. Hence, in the multistage planning, the objective is to minimize the present value of the complete investment carried out throughout the simulated time periods.

Additionally, a solution for the TNEP problem must be a network in which the electric energy is distributed in a safe and reliable way to the electric load centers from the generation sources. Therefore, it is important to guarantee robust networks when a contingency occurs. For that reason, the security criteria must be considered in TNEP studies.
Solution techniques for the TNEP problem have included classical optimization methods, such as the branch and bound algorithm and its specialized versions, and metaheuristics, such as simulated annealing and genetic algorithms. In [1], [3], and [4], the state-of-the-art of the TNEP problem is discussed, and works that consider security constraints and static and multistage planning are listed.

In the specialized literature, it is common that the TNEP problem is solved considering only HVAC lines. However, special attention is being placed on HVDC transmission technologies. The HVDC technologies are divided into two principal categories: the first, based on line-commutated converters (LCC) and the second, based on voltage-source converters (VSC). Both technologies differ in many characteristics: (i) the cost of VSC is 10 to $15 \%$ higher; (ii) the LCC technology has a higher power capability; (iii) the VSC technology can control the reactive power interchange with the AC network; (iv) the LCC technology has a higher reliability; (v) LCC is a more mature technology; (vi) VSC has an insignificant level of harmonic generation. All the characteristics can be found in [5]-[7]. The growing importance of investment in HVDC lines is discussed in [8]. The HVDC lines can be more attractive than HVAC lines, due to the following advantages: (i) when compared to an equivalent HVAC system, the HVDC system can transport a higher amount of power per conductor; (ii) the corridor of the HVDC system is narrower than that of the equivalent HVAC system; (iii) the HVDC system can control the power flow; (iv) when it is considered to connect two asynchronous or synchronous HVAC systems by means of an HVDC system, AC voltage control can be maintained and independently exercised on both $\mathrm{AC}$ systems, i.e., the HVDC system does not impose the maximum angular difference constraints to the model [5], [9]; (v) HVDC systems generate a positive impact on the stability of the system.

There are several feasibility studies that evaluate HVDC links inside HVAC networks. In [8] and [10], HVAC/HVDC comparative studies and HVDC completed projects are presented. Then, with a focus on TNEP studies in which a mathematical model is considered, the HVDC technology in [11] and [12] is included in the transmission planning, without consideration of security constraints or multistage planning. Moreover, in [12], the study is focused on markets; the contingency analysis is stochastic, not exhaustive; and the power losses are neglected.

At present, works that consider the TNEP problem with multistage and/or security constraints using mixed-integer linear modeling can be found in [2], [13], and [14], where the HVDC lines are not included. In [2], the model is designed to allow for the construction of only one line per transmission corridor, so that the authors are forced to reduce the maximum number of lines added in each corridor to one, and they do not consider the possibility of adding lines in all the corridors, reducing the search space considerably. This search space reduction procedure is accomplished without considering any clever strategy; hence, the solutions achieved by the method are feasible, but may not be optimal or of sufficiently good quality. Also, in [2], the cost of losses is not considered. In [13] and [14], strategies to reduce the search space to obtain solutions in a reasonable 
time frame are implemented. With respect to power losses and security criteria in [13] and [14], the losses are neglected, and in [14], security analysis is not included.

This work presents two main contributions. The first contribution considers a mixed-integer linear programming (MILP) model for solving the multistage TNEP problem, including security constraints and a combination of the planning with HVAC and HVDC proposals, to find an expansion plan that meets the requirements of future demand. The proposed MILP model also considers the transmission energy losses in the lines, to obtain a more realistic solution and a future network designed for minimal losses. For this purpose, the losses are penalized and considered as a cost of operation.

Moreover, since the planning horizon is divided into several stages, the investments in lines and the energy loss costs must be discounted back to their present values. Hence, the energy losses are evaluated for each period in the timeline, according to the investment performed in the previous stage, i.e., the operation for a stage considers the topology of the system planned in the previous stage. In addition, for the security analysis, a group of lines (or all the lines in the system) can be selected to consider contingencies, the above for both HVAC and HVDC lines; it is a design criterion of the network planner. Since the transmission of power in HVDC systems using LCC and VSC share some characteristics and this work is based on the DC power flow, it is considered that the HVDC lines do not impose the maximum angular difference constraints to the model. This aspect helps to increase the power transfer in HVAC lines that form closed loops with HVDC lines and can increase the power transfer in new HVAC lines, whose operation is limited by the maximum voltage phase angle difference allowed by other HVAC lines. Also, the model considers the costs according to the technology used, which are based on the study presented in [15]. It should be clarified that the mathematical representation is more adequate for LCC. For a more specific representation of the VSC technology, the model should include the reactive problem. In addition, HVDC lines can be monopolar or bipolar, and the model incorporates this characteristic for the security analysis.

The second contribution proposes a smart search space reduction method (SSRM) for the multistage planning, which considers losses, contingencies, and HVAC/HVDC lines, with the possibility to obtain very high-quality solutions or, in most cases, the optimum solution of the problem in a reduced time. A special feature of the SSRM is that its operating principle can be applied to any planning problem.

The model was implemented using the AMPL modeling language and was solved using the CPLEX commercial solver. The Garver's and Southern Brazilian electrical systems are used to demonstrate the effectiveness of the method. The results obtained demonstrate that the MILP model can evaluate HVDC proposals and show the importance of including HVDC links in the planning process.

The organization of the paper is as follows: Section II shows the mathematical representation of the HVAC and HVDC lines, where the operation and investment variables used are described.

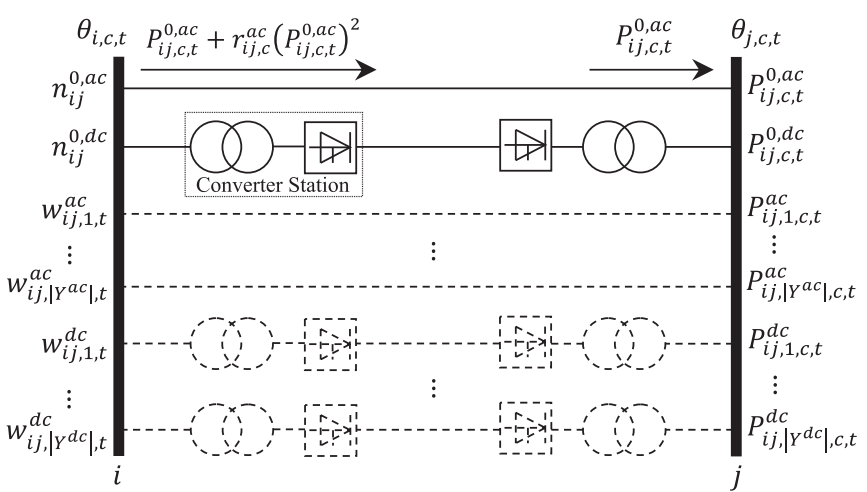

Fig. 1. Line installation options in a transmission corridor.

Also, it presents the objective function and the constraints of the model, and it explains how the contingencies are considered. In addition, due to the nonlinear characteristic of the losses, the explanation of the piecewise linearization of the squared power flow variables is shown. In Section III, the SSRM is introduced. Section IV presents the results, followed by a discussion. Finally, Section V presents the conclusion.

\section{MILP MOdEL FOR THE TNEP PROBLEM}

This section presents the complete MILP model for the multistage HVAC/HVDC TNEP problem, considering contingencies and energy losses.

\section{A. Transmission Line Representation, Power Losses Approximation, and Contingency Aspects}

The proposed MILP model considers $|T|$ stages and $|C|=$ $\left|C^{0}\right|+\left|C^{a c}\right|+\left|C^{d c}\right|$ contingency scenarios, where $|$.$| indi-$ cates the number of elements of a set, i.e., its cardinality. Fig. 1 illustrates the possible line installation options and the power flow variables used. In this paper, it is considered that, for a given corridor, the possible HVAC line options have the same capacities and technical specifications (as in the classic TNEP problem [1]). Options with different characteristics can be easily included in the model; however, they imply a greater computational complexity to solve the problem, since its size increases significantly. The above discussion is also true for HVDC lines. Fig. 1 also shows that it is possible to have installation options of HVAC and HVDC lines at the same corridor $i j$. The existing and each candidate HVAC or HVDC line in corridor $i j$ has a group of variables that describes its model. Since there are scenarios of contingency and stages with different values of forecasted demands and new generation power plants, each scenario in each stage presents a different value for each voltage phase angle and direction and magnitude of the power flow. Thus, a group of variables that characterizes the operation of the system in each scenario in each stage must be defined. In Fig. 1, the group of power flow variables for the candidate HVAC lines in corridor $i j$ is defined by $P_{i j, y, c, t}^{a c}$, where $i j$ represents the corridor, $y$ $\left(y \in Y^{a c}\right)$ represents the line option, $c$ refers to the contingency scenario, and $t$ refers to the stage in the planning horizon. This 
also applies to the other power flow variables presented in Fig. 1. With respect to the investment variables in the new lines, $w_{i j, y, t}^{a c}$ (with $y \in Y^{a c}$ ) and $w_{i j, y, t}^{d c}$ (with $y \in Y^{d c}$ ) are binary variables and represent the installation of a candidate HVAC or HVDC line option $y$ in the corridor $i j$ at stage $t$. If either of the two variables, $w_{i j, y, t}^{a c}$ or $w_{i j, y, t}^{d c}$, takes a value of " 1, " then the new line option $y$ is added; otherwise, a value of " 0 " indicates that the line option $y$ is not selected for construction.

Since the options for the HVAC lines are the same for a corridor $i j$, the model uses the same value of reactance $\left(x_{i j}\right)$ for the existing and each candidate HVAC line in corridor $i j$. Furthermore, each bus $i$ of the network has a variable for the voltage phase angle, $\theta_{i, c, t}$, that has a minimum and maximum value of $-\bar{\theta}$ and $\bar{\theta}$, respectively. The maximum value is used to define the disjunctive parameter " $2 \bar{\theta}$ " [14] and limit the maximum degree of freedom of the voltage phase angle difference in corridor $i j$.

With respect to power energy losses in the HVAC system, an approximation based on [2] is assumed. The current for corridor $i j$ can be calculated using (1).

$$
V_{j} I_{i j}^{a c}=\sqrt{\left(P_{i j}^{a c}\right)^{2}+\left(Q_{i j}^{a c}\right)^{2}}
$$

In this work, only the active problem is considered, and the voltage magnitudes at all buses are considered equal to 1.0 p.u. (DC model), so that from (1), $I_{i j}^{a c}=\left|P_{i j}^{a c}\right|$. Therefore, the power flow $P_{i j}^{a c}$ is used to calculate the power losses in corridor $i j$, instead of the current $I_{i j}^{a c}$.

For the security analysis, two matrices, $N C^{a c}$ and $N C^{d c}$, are defined, in which each row indicates a corridor and each column a contingency scenario, with dimensions $\left|\Omega_{a c}\right| \times\left|C^{a c}\right|$ and $\left|\Omega_{d c}\right| \times\left|C^{d c}\right|$, correspondingly. The sets $C^{a c}$ and $C^{d c}$ contain the selected group of contingencies for HVAC and HVDC lines. In the columns, only the entry corresponding to the line under contingency is equal to one, while the other elements remain at zero.

The model must consider the following scenarios of operation: (i) normal operation, (ii) HVAC line contingencies, and (iii) HVDC line contingencies. The aim is to develop constraints for each possible scenario of contingency. Hence, two sets must be defined, $C I$ and $C I I$, that describe the following two scenarios, respectively: (i) there is a contingency in one line in a corridor with at least one existing line, and (ii) there is a contingency in a corridor where there are no existing lines, meaning that the contingency will be considered in a future possible line. The aim is that a contingency in a line belonging either to set $C^{a c}$ or $C^{d c}$ can be defined as belonging either to $C I$ or $C I I$, and that depending on the type of the contingency, the constraint type will be introduced in the model.

\section{B. Objective Function}

In this work, the objective function is to minimize the cost of the investments in lines and the cost of operation, which depends on the energy losses. Since multistage planning is considered, the planning horizon is divided in several stages, and the investment cost $I C_{t}$ for each stage $t$ must be discounted back to its present value. Fig. 2(a) shows a planning horizon with three

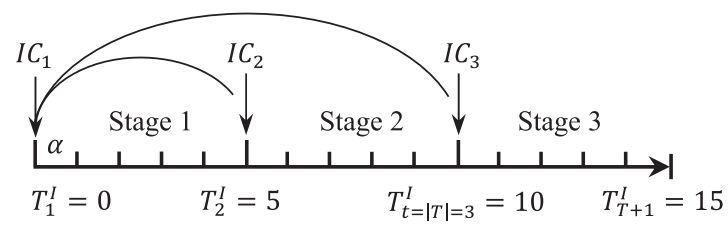

(a)

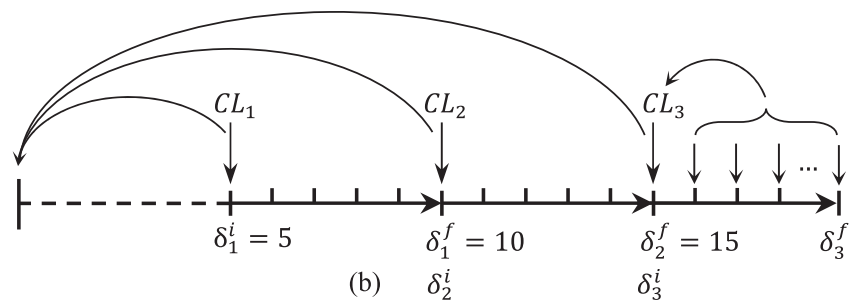

Fig. 2. (a) Planning and (b) operation timelines.

stages of investment, where $\alpha$, the annual discount factor to find the present investment value, is assumed. It is considered that the investment is performed at the beginning of each stage. The calculation of the energy losses cost $\left(C L_{t}\right)$ for the operation at stage $t$ considers the topology of the system planned in the corresponding investment stage $t$. Fig. 2(b) shows the operation timeline, where $C L_{t}$ is the discounted value for the operation at stage $t$, which corresponds to the time interval from $\delta_{t}^{i}$ to $\delta_{t}^{f}$. In other words, the cost of losses for the time interval between years $\delta_{1}^{i}=5$ and $\delta_{1}^{f}=10$ is calculated for the investment performed in the year $T_{1}^{I}=0$, and so on. This agrees with reality, where the investment is made first, and years later, when the construction is finished, the installed lines begin the operation at the designed stage.

The investment and energy losses costs must be discounted back to their present value, as shown in (2)

$$
\min v=\sum_{t \in T} \frac{I C_{t}}{(1+\alpha)^{T_{t}^{I}}}+\frac{C L_{t}}{(1+\alpha)^{\delta_{t}^{i}}}
$$

where $I C_{t}$ is shown in (3) for the first stage and in (4) for $t>1$.

$$
\begin{aligned}
I C_{t \mid t=1}= & \sum_{i j \in \Omega_{a c}} \sum_{y \in Y^{a c}} C_{i j}^{a c} w_{i j, y, 1}^{a c}+\sum_{i j \in \Omega_{d c}} \sum_{y \in Y^{d c}} C_{i j}^{d c} w_{i j, y, 1}^{d c} \\
I C_{t \mid t>1}= & \sum_{i j \in \Omega_{a c}} \sum_{y \in Y^{a c}} C_{i j}^{a c}\left(w_{i j, y, t}^{a c}-w_{i j, y, t-1}^{a c}\right) \\
& +\sum_{i j \in \Omega_{d c}} \sum_{y \in Y^{d c}} C_{i j}^{d c}\left(w_{i j, y, t}^{d c}-w_{i j, y, t-1}^{d c}\right)
\end{aligned}
$$

The values of $C L_{t}$ for the period $\left[\delta_{t}^{i}, \delta_{t}^{f}\right]$, discounted back to the end of the operation at stage $t$, are shown in (5) and (6) [16]. 
Constant demand is assumed during an operation period.

$$
\begin{aligned}
C L_{t} & =\left(\frac{\lambda_{t} S^{b} C^{e}}{10^{6}}\right)\left[\frac{(1+\alpha)^{\delta_{t}^{f}-\delta_{t}^{i}}-1}{\left.\alpha(1+\alpha)^{\delta_{t}^{f}-\delta_{t}^{i}}\right]}\right. \\
& \times\left(\Delta_{0} P_{0, t}^{l s}+\sum_{c \in C^{a c}} \Delta_{c}^{a c} P_{c, t}^{l s}+\sum_{c \in C^{d c}} \Delta_{c}^{d c} P_{c, t}^{l s}\right) \\
P_{c, t}^{l s} & =\sum_{i j \in \Omega_{a c}}\left[r_{i j, c}^{a c}\left(P_{i j, c, t}^{0, a c}\right)^{2}+\sum_{y \in Y^{a c}} r_{i j, y, c}^{a c}\left(P_{i j, y, c, t}^{a c}\right)^{2}\right] \\
& +\sum_{i j \in \Omega_{d c}}\left[r_{i j, c}^{d c}\left(P_{i j, c, t}^{0, d c}\right)^{2}+\sum_{y \in Y^{d c}} r_{i j, y, c}^{d c}\left(P_{i j, y, c, t}^{d c}\right)^{2}\right]
\end{aligned}
$$

In (5), $P_{c . t}^{l s}$ is the value of the power losses and depends on the contingency scenario $c$ and stage $t ; \lambda_{t}$ is the load factor on stage $t$, and $\Delta_{c}^{a c}$ and $\Delta_{c}^{d c}$ are the annual system operation times in scenario $c$ for contingencies in HVAC and HVDC lines, where the time duration of normal operation $\Delta_{0}$ is much greater than the operation times under contingencies. The model includes the minimization of the losses under contingency scenarios, so that the linearization function presented in Section II-D can be used. Note that, since the operation times under contingency scenarios are small, the losses under contingency almost do not influence the objective function.

\section{Constraints}

The constraints of the proposed MILP model are presented in this section. Equation (7) represents the active power balance at bus $i$, at stage $t$, and in scenario $c$, where the power loss influence is included. In (8)-(10) the limits of the active power generation and voltage phase angle are presented. Constraint (11) limits the number of lines allowed in each HVAC corridor.

$$
\begin{aligned}
\sum_{k i \in \Omega_{a c}} & \left(P_{k i, c, t}^{0, a c}+\sum_{y \in Y^{a c}} P_{k i, y, c, t}^{a c}\right) \\
+ & \sum_{k i \in \Omega_{d c}}\left(P_{k i, c, t}^{0, d c}+\sum_{y \in Y^{d c}} P_{k i, y, c, t}^{d c}\right) \\
& -\sum_{i j \in \Omega_{a c}}\left[P_{i j, c, t}^{0, a c}+r_{i j, c}^{a c}\left(P_{i j, c, t}^{0, a c}\right)^{2}\right. \\
+ & \left.\sum_{y \in Y^{a c}}\left[P_{i j, y, c, t}^{a c}+r_{i j, y, c}^{a c}\left(P_{i j, y, c, t}^{a c}\right)^{2}\right]\right] \\
- & \sum_{i j \in \Omega_{d c}}\left[P_{i j, c, t}^{0, d c}+r_{i j, c}^{d c}\left(P_{i j, c, t}^{0, d c}\right)^{2}\right.
\end{aligned}
$$

$$
\begin{gathered}
\left.+\sum_{y \in Y^{d c}}\left[P_{i j, y, c, t}^{d c}+r_{i j, y, c}^{d c}\left(P_{i j, y, c, t}^{d c}\right)^{2}\right]\right] \\
+g_{i, c, t}-d_{i, t}=0 \quad \forall i \in \Omega_{B}, \forall c \in C, \forall t \in T \\
0 \leq g_{i, c, t} \leq \bar{g}_{i, t} \quad \forall i \in \Omega_{B}, \forall c \in C, \forall t \in T \\
-\bar{\theta} \leq \theta_{i, c, t} \leq \bar{\theta} \quad \forall i \in \Omega_{B}, \forall c \in C, \forall t \in T \\
\theta_{i, c, t}=0 \quad i=S l a c k, \forall c \in C, \forall t \in T \\
\sum_{y \in Y^{a c}} w_{i j, y, t}^{a c} \leq \bar{n}_{i j}^{a c} \quad \forall i j \in \Omega_{a c}, \forall t \in T .
\end{gathered}
$$

Constraint (12) establishes a sequence to install lines in each corridor. Suppose the analysis is made for a given HVAC corridor $i j$. The first line that should be added is $w_{i j, 1, t}^{a c}=1$, so that the line corresponding to $w_{i j, 2, t}^{a c}=1$ cannot be added unless the line corresponding to $w_{i j, 1, t}^{a c}$ was added first. Hence, the solution method applied becomes more efficient, since the investment sequence reduces the number of combinations that must be analyzed in a corridor. On the other hand, if corridor $i j$ is under contingency in scenario $c$, and if a line is needed in the solution in this corridor, (12) forces the building of the first line with contingency, even if it will not operate in this scenario; then, if necessary, other lines are installed. Constraint (13) states that the number of HVAC lines in corridor $i j$ at stage $t$ must be greater than or equal to the number of lines in the same corridor at stage $t-1$, i.e., lines cannot be removed from one stage to the next

$$
\begin{aligned}
w_{i j, y, t}^{a c} \leq w_{i j, y-1, t}^{a c} \quad \forall i j & \in \Omega_{a c}, \forall y \in Y^{a c} \mid y>1, \\
\forall t & \in T \\
w_{i j, y, t-1}^{a c} \leq w_{i j, y, t}^{a c} \quad \forall i j & \in \Omega_{a c}, \forall y \in Y^{a c}, \\
\forall t & \in T \mid t>1
\end{aligned}
$$

In (14)-(16), constraints with the same function as (11)-(13) are presented, but in this case, for candidate HVDC lines.

$$
\begin{aligned}
& \sum_{y \in Y^{d c}} w_{i j, y, t}^{d c} \leq \bar{n}_{i j}^{d c} \quad \forall i j \in \Omega_{d c}, \forall t \in T \\
& w_{i j, y, t}^{d c} \leq w_{i j, y-1, t}^{d c} \quad \forall i j \in \Omega_{d c}, \forall y \in Y^{d c} \mid y>1, \forall t \in T \\
& w_{i j, y, t-1}^{d c} \leq w_{i j, y, t}^{d c} \quad \forall i j \in \Omega_{d c}, \forall y \in Y^{d c}, \forall t \in T \mid t>1 .
\end{aligned}
$$

The equations shown operate in both normal and contingency scenarios. Kirchhoff's voltage law in existing and candidate HVAC networks, with their limits and the equations of HVDC systems, depend on the operative state. Hence, Kirchhoff's voltage law must be satisfied for the existing HVAC network, as 
shown in (17) and (18).

$$
\begin{array}{r}
P_{i j, c, t}^{0, a c}-\frac{n_{i j}^{0, a c}\left(\theta_{i, c, t}-\theta_{j, c, t}\right)}{x_{i j}}=0 \\
\left(\forall i j \in \Omega_{a c}, \forall c \in C^{0}, \forall t \in T\right) \cup \\
\left(\forall i j \in \Omega_{a c}, \forall c \in C^{a c} \mid c \in C I I, \forall t \in T\right) \cup \\
\left(\forall i j \in \Omega_{a c}, \forall c \in C^{d c}, \forall t \in T\right) \\
P_{i j, c, t}^{0, a c}-\frac{\left(n_{i j}^{0, a c}-N C_{i j, c}^{a c}\right)\left(\theta_{i, c, t}-\theta_{j, c, t}\right)}{x_{i j}}=0 \\
\forall i j \in \Omega_{a c}, \forall c \in C^{a c} \mid c \in C I, \forall t \in T
\end{array}
$$

In (18), $N C_{i j, c}^{a c}$ is used to simulate the security criteria if the contingency scenario $c$ is in an existing line. Note that (17) and (18) complement each other; if the contingency scenario $c$ belongs to case $C I$ (contingency in an existing line), then (18) is applied. Otherwise, (17) is applied in the other cases: normal operation, contingency in new HVAC lines, and contingency in HVDC lines (i.e., a fault in the HVDC network should not affect the mathematical representation of the constraints on the HVAC lines), for each stage.

The limits of the power transfer in existing HVAC corridors must be satisfied, as shown in (19) and (20)

$$
\begin{array}{r}
\left|P_{i j, c, t}^{0, a c}\right|+r_{i j, c}^{a c}\left(P_{i j, c, t}^{0, a c}\right)^{2} \leq n_{i j}^{0, a c} \bar{P}_{i j}^{a c} \\
\left(\forall i j \in \Omega_{a c}, \forall c \in C^{0}, \forall t \in T\right) \cup \\
\left(\forall i j \in \Omega_{a c}, \forall c \in C^{a c} \mid c \in C I I, \forall t \in T\right) \cup \\
\left(\forall i j \in \Omega_{a c}, \forall c \in C^{d c}, \forall t \in T\right) \\
\left|P_{i j, c, t}^{0, a c}\right|+r_{i j, c}^{a c}\left(P_{i j, c, t}^{0, a c}\right)^{2} \leq\left(n_{i j}^{0, a c}-N C_{i j, c}^{a c}\right) \bar{P}_{i j}^{a c} \\
\forall i j \in \Omega_{a c}, \forall c \in C^{a c} \mid c \in C I, \forall t \in T .
\end{array}
$$

Constraints (19) and (20) also complement each other, i.e., if the contingency scenario $c$ belongs to case $C I$, then (20) is applied; otherwise, (19) is applied, as in (17) and (18). Note that in (19) and (20), the power losses are considered.

For the candidate HVAC network, Kirchhoff's voltage law must be satisfied, as shown in (21) and (22).

$$
\begin{gathered}
\left|x_{i j} P_{i j, y, c, t}^{a c}-\left(\theta_{i, c, t}-\theta_{j, c, t}\right)\right| \leq 2 \bar{\theta}\left(1-w_{i j, y, t}^{a c}\right) \\
\left(\forall i j \in \Omega_{a c}, \forall y \in Y^{a c}, \forall c \in C^{0}, \forall t \in T\right) \cup \\
\left(\forall i j \in \Omega_{a c}, \forall y \in Y^{a c}, \forall c \in C^{a c} \mid c \in C I, \forall t \in T\right) \cup \\
\left(\forall i j \in \Omega_{a c}, \forall y \in Y^{a c}\left|y>1, \forall c \in C^{a c}\right| c \in C I I, \forall t \in T\right) \\
\cup\left(\forall i j \in \Omega_{a c}, \forall y \in Y^{a c}, \forall c \in C^{d c}, \forall t \in T\right) \\
\left|x_{i j} P_{i j, y, c, t}^{a c}-\left(\theta_{i, c, t}-\theta_{j, c, t}\right)\right| \leq 2 \bar{\theta}\left[1-w_{i j, y, t}^{a c}\left(1-N C_{i j, c}^{a c}\right)\right] \\
\forall i j \in \Omega_{a c}, \forall y \in Y^{a c}\left|y=1, \forall c \in C^{a c}\right| c \in C I I, \forall t \in T \quad(22)
\end{gathered}
$$

Again, constraints (21) and (22) complement each other. If the contingency scenario $c$ belongs to case $C I I$ and the line analyzed is the first option (a contingency in the candidate line $y=1)$, (22) is applied; otherwise, (21) is applied. This means that, if a contingency is considered in a corridor with no existing lines, it will always be in the first candidate line of that corridor, i.e., these constraints simulate a contingency in a possible future HVAC line, with the help of $N C_{i j, c}^{a c}$. Furthermore, (21) and (22) are "activated" when the decision variable $w_{i j, y, t}^{a c}$ takes the value of 1 . The parameter " $2 \bar{\theta}$ " works as a "big-M factor" and provides a sufficient degree of freedom for the voltage phase angle difference between buses of corridors without lines. This means that constraints (21) and (22) become inactive for corridors that do not have lines, which makes the effect of eliminating such constraints [14].

The limits in the power transfer in candidate HVAC lines must be satisfied, as shown in (23) and (24). Constraints (23) and (24) complement each other, as in (21) and (22), for candidate lines. If the contingency scenario $c$ belongs to case $C I I$ and the line analyzed is $y=1$, then (24) is applied; otherwise, (23) is applied. Note that, in (23) and (24), the power losses in candidate lines are considered

$$
\begin{gathered}
\left|P_{i j, y, c, t}^{a c}\right|+r_{i j, y, c}^{a c}\left(P_{i j, y, c, t}^{a c}\right)^{2} \leq w_{i j, y, t}^{a c} \bar{P}_{i j}^{a c} \\
\left(\forall i j \in \Omega_{a c}, \forall y \in Y^{a c}, \forall c \in C^{0}, \forall t \in T\right) \cup \\
\left(\forall i j \in \Omega_{a c}, \forall y \in Y^{a c}, \forall c \in C^{a c} \mid c \in C I, \forall t \in T\right) \cup \\
\left(\forall i j \in \Omega_{a c}, \forall y \in Y^{a c}\left|y>1, \forall c \in C^{a c}\right| c \in C I I, \forall t \in T\right) \\
\cup\left(\forall i j \in \Omega_{a c}, \forall y \in Y^{a c}, \forall c \in C^{d c}, \forall t \in T\right) \\
\left|P_{i j, y, c, t}^{a c}\right|+r_{i j, y, c}^{a c}\left(P_{i j, y, c, t}^{a c}\right)^{2} \leq w_{i j, y, t}^{a c} \bar{P}_{i j}^{a c}\left(1-N C_{i j, c}^{a c}\right) \\
\forall i j \in \Omega_{a c}, \forall y \in Y^{a c}\left|y=1, \forall c \in C^{a c}\right| c \in C I I, \forall t \in T .
\end{gathered}
$$

Finally, the equations of the HVDC systems must be defined. In this work, an HVDC line is modeled as a link that carries active power within its power limits as a function of the existing network and possible investments. The operation of each converter station belongs to the short-term operation planning. The direction and magnitude of the power flow is determined in the solution process, without exceeding the capacity limits. The model of the HVDC lines must satisfy only Kirchhoff's current law, whereas Kirchhoff's voltage law is not considered, because it is not constrained by the voltage phase angles at both AC buses at the HVDC line terminals [5]. Kirchhoff's current law is considered in the active power balance at bus $i$, stage $t$, and scenario $c$, as presented in (7). Hence, the constraints to limit the power flow in HVDC corridors, in terms of the existing HVDC network $\left(n_{i j}^{0, d c}\right)$ and the investment variables $\left(w_{i j, y, t}^{d c}\right)$ in the candidate HVDC network, are represented in (25)-(28)

$$
\begin{gathered}
\left|P_{i j, c, t}^{0, d c}\right|+r_{i j, c}^{d c}\left(P_{i j, c, t}^{0, d c}\right)^{2} \leq n_{i j}^{0, d c} \bar{P}_{i j}^{d c} \\
\left(\forall i j \in \Omega_{d c}, \forall c \in C^{0}, \forall t \in T\right) \cup \\
\left(\forall i j \in \Omega_{d c}, \forall c \in C^{d c} \mid c \in C I I, \forall t \in T\right) \cup \\
\left(\forall i j \in \Omega_{d c}, \forall c \in C^{a c}, \forall t \in T\right)
\end{gathered}
$$




$$
\begin{gathered}
\left|P_{i j, c, t}^{0, d c}\right|+r_{i j, c}^{d c}\left(P_{i j, c, t}^{0, d c}\right)^{2} \leq\left(n_{i j}^{0, d c}-N C_{i j, c}^{d c} / L C_{i j}\right) \bar{P}_{i j}^{d c} \\
\forall i j \in \Omega_{d c}, \forall c \in C^{d c} \mid c \in C I, \forall t \in T \quad \text { (26) } \\
\left|P_{i j, y, c, t}^{d c}\right|+r_{i j, y, c}^{d c}\left(P_{i j, y, c, t}^{d c}\right)^{2} \leq w_{i j, y, t}^{d c} \bar{P}_{i j}^{d c} \\
\left(\forall i j \in \Omega_{d c}, \forall y \in Y^{d c}, \forall c \in C^{0}, \forall t \in T\right) \cup \\
\left(\forall i j \in \Omega_{d c}, \forall y \in Y^{d c}, \forall c \in C^{d c} \mid c \in C I, \forall t \in T\right) \cup \\
\left(\forall i j \in \Omega_{d c}, \forall y \in Y^{d c}\left|y>1, \forall c \in C^{d c}\right| c \in C I I, \forall t \in T\right) \\
\cup\left(\forall i j \in \Omega_{d c}, \forall y \in Y^{d c}, \forall c \in C^{a c}, \forall t \in T\right) \quad(27) \\
\left|P_{i j, y, c, t}^{d c}\right|+r_{i j, y, c}^{d c}\left(P_{i j, y, c, t}^{d c}\right)^{2} \leq w_{i j, y, t}^{d c}\left(1-N C_{i j, c}^{d c} / L C_{i j}\right) \bar{P}_{i j}^{d c} \\
\forall i j \in \Omega_{d c}, \forall y \in Y^{d c}\left|y=1, \forall c \in C^{d c}\right| c \in C I I, \forall t \in T .
\end{gathered}
$$

Similar to the HVAC cases, constraints (25) and (26) complement each other for the existing HVDC network, while (27) and (28) are defined for the candidate HVDC network. In (26) and (28), $L C_{i j}$ is the loss of line capacity. If the HVDC system is monopolar, then $L C_{i j}=1$, and if the HVDC system is bipolar, then $L C_{i j}=2$, i.e., for a contingency in a bipolar line, only one pole is lost. This is because, in a bipolar line, each pole works independently; also, from the reliability point of view, an electrical contingency in one pole is assumed to not affect the other pole [5], [17].

\section{Linearization of the Square of the Power Flow}

To develop an MILP model for the TNEP problem, some equations presented are modified in this section. Recall that the term (6) of the objective function and constraints (7), (19), (20), (23), (24), and (25)-(28), related to the quadratic power flow variables, are nonlinear. The quadratic terms can be represented using the piecewise linear functions (29)-(32)

$$
\begin{aligned}
\left(P_{i j, c, t}^{0, a c}\right)^{2} & \approx f\left(P_{i j, c, t}^{0, a c}, \bar{P}_{i j}^{a c}, L\right) \quad \forall i j \in \Omega_{a c}, \\
\forall c & \in C, \forall t \in T \\
\left(P_{i j, c, t}^{0, d c}\right)^{2} & \approx f\left(P_{i j, c, t}^{0, d c}, \bar{P}_{i j}^{d c}, L\right) \quad \forall i j \in \Omega_{d c}, \\
\forall c & \in C, \forall t \in T \\
\left(P_{i j, y, c, t}^{a c}\right)^{2} & \approx f\left(P_{i j, y, c, t}^{a c}, \bar{P}_{i j}^{a c}, L\right) \\
\forall i j & \in \Omega_{a c}, \forall y \in Y^{a c}, \\
\forall c & \in C, \forall t \in T \\
\left(P_{i j, y, c, t}^{d c}\right)^{2} & \approx f\left(P_{i j, y, c, t}^{d c}, \bar{P}_{i j}^{d c}, L\right) \\
\forall i j & \in \Omega_{d c}, \forall y \in Y^{d c}, \\
\forall c \in C, \forall t & \in T .
\end{aligned}
$$

The piecewise linear approximation function $f(z, \bar{z}, L)$ used in (29)-(32) is defined in (33)-(38), based on [2], [18].

$$
\begin{array}{rlr}
f(z, \bar{z}, L) & =\sum_{l=1}^{L} S l_{z, l} \Delta_{z, l} & \\
z & =z^{+}-z^{-} & \\
z^{+}+z^{-} & =\sum_{l=1}^{L} \Delta_{z, l} & \\
0 & \leq \Delta_{z, l} \leq \bar{z} / L \quad l=1 \ldots L \\
S l_{z, l} & =(2 l-1) \bar{z} / L & l=1 \ldots L \\
z^{+}, z^{-} & \geq 0 &
\end{array}
$$

Equation (33) is the square-value linear approximation of variable $z$ by using variables $\Delta_{z, l}$ with the aim of discretizing the absolute value of $z$. The variables $\Delta_{z, l}$ define the length of each discretized segment whose sum is equal to $|z|$. The auxiliary variables $z^{+}$and $z^{-}$model $|z|$, as is shown in (34) and (35). The parameter $S l_{z, l}$ represents the slope of the $l$ th line segment of the linearization process and is used to evaluate the contribution of $\Delta_{z, l}$ in each step of the discretization. The upperbound $\bar{z} / L$ represents the length of each discretized segment where $\bar{z}$ is the maximum value of $z$. Then, each quadratic term in the model can be approximated by (33)-(38); consequently, the number of constraints and continuous variables in the model is incremented. Since the objective function of the problem minimizes the losses in all operation scenarios, the adjacency of the discretized segments is ensured, i.e., the segments $\Delta_{z, l}$ with smaller values of $l$ are completed first, because otherwise, the right-hand side of (33) would increase, leading to higher values of the objective function [2].

\section{Search Space Reduction Method}

The proposed search space reduction method is based on [19], where the solution of the North-Northeastern Brazilian system was improved in terms of time with respect to [14], which reported the best-known solution for this system. The objective is to identify corridors in the system to decrease their maximum number of new lines allowed, to reduce the search space of the problem. To accomplish this, two tools were developed. The aim of the first tool (partitioning stage) is to separate the complete transmission system into $m$ subsystems to analyze each one with the proposed MILP model (the three techniques to separate the system will be shown later). Consequently, however, each subsystem can have insufficient generation to meet the demand, due to the removal of lines where active power flow existed. To overcome this problem, the second tool is used (injection stage). It is based on an injection of active power in the boundary of each subsystem in the form of generation (in subsystems that need active power injection) or in the form of demand (in subsystems that provide active power to another subsystem). These injections are done on buses that were connected by the removed lines. Then, to interconnect the partitioning and injection stages, each subsystem can be represented by a demand-bus 
or generation-bus, after the execution of a power balance has taken place in each subsystem. Then, an equivalent system can be built with the removed lines and considering each subsystem as an equivalent bus. The subsystems obtained from the partitioning stage and the equivalent system of the injection stage are solved iteratively using the proposed MILP model with some modifications, in order to obtain new limits of line additions in each corridor.

In contrast to [19], in this work, contingencies, HVDC lines, and power losses are included. The challenge of including losses is that they are unknown, because the future transmission network is uncertain, yet they must be considered in the power balance of each subsystem. Furthermore, losses change with each contingency scenario. In this paper, a method to calculate the approximate losses in each subsystem from the partitioning stage is proposed. The techniques to partition the system, injection of active power approach, method to approximate the power losses, and complete method to reduce the search space are shown below. Garver's 6-bus system [see Fig. 3(a)] is used to illustrate the partitioning methods.

\section{A. Partitioning Stage}

1) Partition based on connection degree: In the geographical representation of a system, zones where the interconnection between buses is strong and other zones, where the interconnection between buses is not very strong, i.e., there are not many lines that interfere with the division of the network, can be identified and separated from the system by removing a minor number of lines to form the subsystems. An illustrative example for the partition based on connection degree is shown in Fig. 3(b), in which two subsystems, one with four buses and the other with two buses, are created. The idea is to divide the system into several subsystems (at least two), minimizing the number of lines removed.

2) Partition based on balance between generation and demand: The system is divided to obtain subsystems so that the generation of active power is enough to meet the demand, trying to avoid the interchange of power between subsystems. Fig. 3(c) presents an example for this type of partition, in which the system is divided into three subsystems, where bus one supplies its own demand, bus three meets the demand of bus five, and bus six meets the demand of buses two and four, with lower values of load shedding.

3) Partition based on minimum effort network: This procedure modifies the complete system by adding a great number of lines in all the corridors. Lines should be added in each corridor until no significant change in the power flows is observed. This emulates an ideal electrical network in which the active power is not forced to flow through specific lines, but through the route of minimum effort. Since many lines are added in this procedure, the contingency analysis does not have influence. Then, the proposed MILP model is used, but only the normal operation scenario is analyzed, for the last stage of the planning. After the model is applied, the magnitude of the power flow is known at each line in all the corridors, and a list that contains the power flows through the corridors can be created (the power flow through a corridor is the sum of the power flow at each line in the corridor). The list can be sorted in ascending order to determine what corridors have the lower magnitude of power flow. Hence, the relative importance of each corridor with respect to the others can be determined, and the system can be divided. The selected corridors to be removed are the ones with the lower value of power flow, i.e., the ones that appear in the first positions on the list. With respect to the MILP model applied in this section, the solution will not add a new line, since a great number of lines was already added to the network; only the minimization of the power losses is carried out. Fig. 3(d) presents an example for this type of partition scheme, where the system is divided in two subsystems, and lines 1-4, 1-6, 4-6, 2-3, 2-5, and 3-5 have the higher values of power flow among all lines in the system when the procedure is applied.

\section{B. Approximation of the Power Losses in a Subsystem}

To find the power losses in a subsystem from the partitioning stage, the MILP model with objective function (39), subject to (8)-(38) and (40)-(41), is applied. Since a subsystem can present a deficit in generation to meet the demand and the power losses, a temporary change in the MILP model presented in Section II is performed, i.e., a load shedding, $\varphi_{i, c, t}$, is added to the left-hand side of (7) as a new variable, with its limits; this produces the model (39)-(41)

$$
\min v_{p}=v+\sigma \sum_{i \in \Omega_{B}} \sum_{c \in C} \sum_{t \in T} \varphi_{i, c, t} .
$$

Subject to: (8)-(38)

$$
\begin{array}{ll}
\text { LHS of }(7)+\varphi_{i, c, t}=0 & \forall i \in \Omega_{B}, \forall c \in C, \forall t \in T \\
0 \leq \varphi_{i, c, t} \leq d_{i, t} & \forall i \in \Omega_{B}, \forall c \in C, \forall t \in T
\end{array}
$$

where the original objective function (2) is replaced by (39) and the original power balance (7) becomes (40). The parameter $\sigma$ is the cost for load shedding. The value of the losses selected to perform the power balance for the injection stage is the one for the contingency scenario $c$ with the highest value.

\section{Injection Stage}

With the values of demand, generation, and approximate losses in each subsystem known (from procedure b), a power balance can be developed for each subsystem to form the equivalent system. The equivalent system is formed by demand and generation buses (each bus representing one subsystem, and the type of the bus depending on the result of the power balance in the subsystem), where these buses are interconnected by the removed lines. As an illustrative example, for the system shown in Fig. 3(b), the equivalent system is formed by two "equivalent buses," the first one formed by buses 1, 2, 3, and 5 and the second one formed by buses 4 and 6 . The equivalent buses are connected by the removed lines. Then, similar to item 3 ) of the partitioning stage, the procedure modifies the equivalent system by adding a great number of reinforcements in the removed corridors. Again, the contingencies do not have influence in a system with many lines in all the corridors. The proposed MILP 


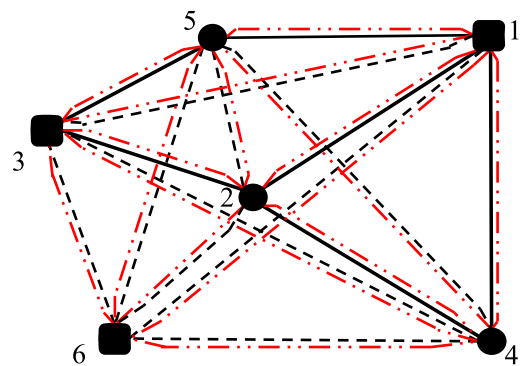

(a)

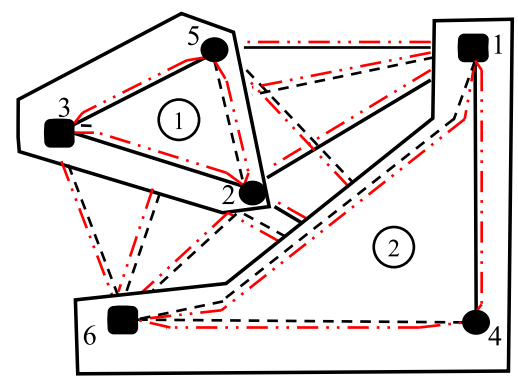

(d)

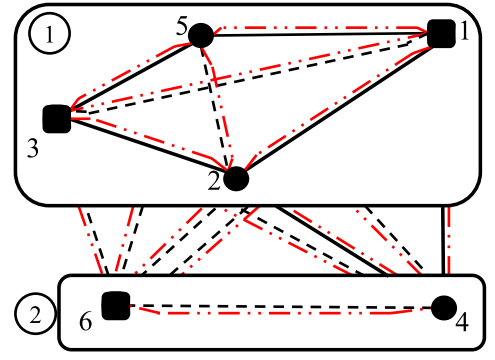

(b)

\begin{tabular}{|c|c|c|c|}
\hline \multicolumn{2}{|c|}{$\begin{array}{l}\text { With candidate } \\
\text { HVDC lines }\end{array}$} & \multicolumn{2}{|c|}{$\begin{array}{l}\text { Without candidate } \\
\text { HVDC lines }\end{array}$} \\
\hline $\bar{n}_{15}^{a c}=3$ & $\bar{n}_{46}^{a c}=3$ & $\bar{n}_{12}^{a c}=2$ & $\bar{n}_{23}^{a c}=3$ \\
\hline $\bar{n}_{23}^{a c}=3$ & $\bar{n}_{23}^{d c}=2$ & $\bar{n}_{14}^{a c}=1$ & $\bar{n}_{26}^{a c}=4$ \\
\hline $\bar{n}_{26}^{a c}=1$ & $\bar{n}_{26}^{d c}=2$ & $\bar{n}_{15}^{a c}=3$ & $\bar{n}_{35}^{a c}=3$ \\
\hline $\begin{array}{l}\bar{n}_{35}^{a c}=3 \\
\bar{n}^{a c}=4\end{array}$ & $\begin{array}{l}\bar{n}_{35}^{d c}=2 \\
\bar{n}_{2 c}^{d c}=2\end{array}$ & $\bar{n}_{16}^{a c}=2$ & $\bar{n}_{46}^{a c}=3$ \\
\hline $\bar{n}_{36}^{a c}=4$ & $\bar{n}_{36}^{d c}=2$ & $\bar{n}_{34}^{a c}=4$ & $\bar{n}_{36}^{a c}=4$ \\
\hline $\bar{n}_{45}^{a c}=4$ & $\bar{n}_{45}^{d c}=2$ & $\bar{n}_{45}^{a c}=4$ & \\
\hline
\end{tabular}

(e)

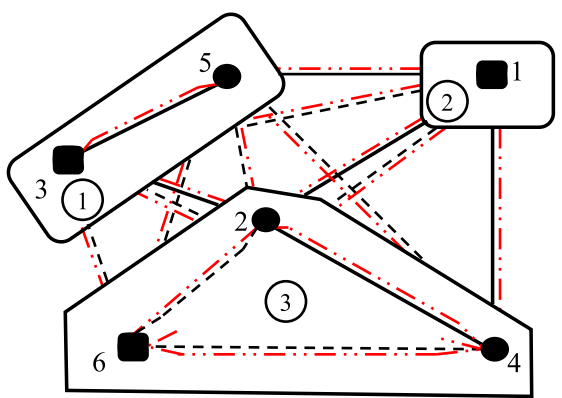

(c)

Fig. 3. (a) Garver's 6-bus system. (b)-(d) Subsystems for each partitioning method. (e) SSRM solution.

model is then applied (for the normal operation scenario and the last stage of the planning) in the equivalent system to find the direction and magnitude of the power flows in the removed lines. Finally, the power balance can be updated according to the values of the power flow entering or leaving each bus of the equivalent system. This update is performed by using the power flow entering in a subsystem as new incoming generation in the corresponding buses of that subsystem and using the power flow leaving a subsystem as new demand in the corresponding buses of that subsystem.

\section{Complete Method}

The method is shown in the flowchart of Fig. 4, where, for each partitioning method, the approximation of the power losses and injection stages are applied iteratively. The aim is to update the injected power at the boundary buses that share corridors with other subsystems. This produces a variation in the power losses, but considering the information from the other subsystems, the injected active power and losses in each subsystem are adjusted until the load shedding is reduced to zero. At the end of this process, the subsystems will have no load shedding, and since the proposed MILP model is applied, the corridors that are part of the solution in each subsystem are classified as having the maximum number of candidate lines reduced to the number of lines installed.

The last step is to build and to solve the proposed MILP model for the complete network with the new limits of line additions, $\bar{n}_{i j}^{a c}$ and $\bar{n}_{i j}^{d c}$. Hence, for each corridor, the maximum limit of additions is selected from the results of all the partitioning methods: the solution with the greatest number of lines is selected for each corridor. Finally, if a corridor is never analyzed inside of a subsystem, it will take the maximum limit of line additions allowed. In the example of Fig. 3, since corridors 3-4, 3-6, 4-5, and 5-6 do not belong to any subsystem, considering the three partitioning methods, their maximum limits of line additions are not changed.

\section{TESTS AND RESUlts}

Garver's 6-bus system and a modified Southern Brazilian system (see Fig. 5) are used to show the precision and efficiency of the proposed method. The test systems share some assumptions, as follows: (i) a planning horizon of fifteen years, divided into three planning stages, as shown in Fig. 2, is considered, with five years per stage; (ii) a sufficient number of blocks for the piecewise linearization is $L=10$ [2]; (iii) The annual discount rate is $\alpha=10 \%$; (iv) the load factor is $\lambda_{t}=0.65$ for all stages; (v) the annual time duration is $8760 \mathrm{~h}$, while the annual duration of each contingency scenario is five hours. The proposed MILP model and the search space reduction method were implemented using the AMPL mathematical modeling language and solved using the CPLEX commercial solver for optimization problems (with default settings) on a computer with a $3.40 \mathrm{GHz}$ Intel Core i7-4770 processor and 16 GB of RAM. The results are shown in the following subsections.

\section{A. Garver's 6-Bus System}

The complete data for Garver's 6-bus, 15-corridor system [see Fig. 3(a)] is available in [20]. The bipolar HVDC lines can be installed in all the same corridors for the HVAC lines. In this system, the data for HVAC and HVDC lines are based on [11], [12], and [15]. In contrast to [11], where the capacity of an HVDC line in a corridor is as high as 2.8 times the capacity of an HVAC line in the same corridor, in this work, the HVAC and HVDC lines in the same corridor have the same capacity, to 


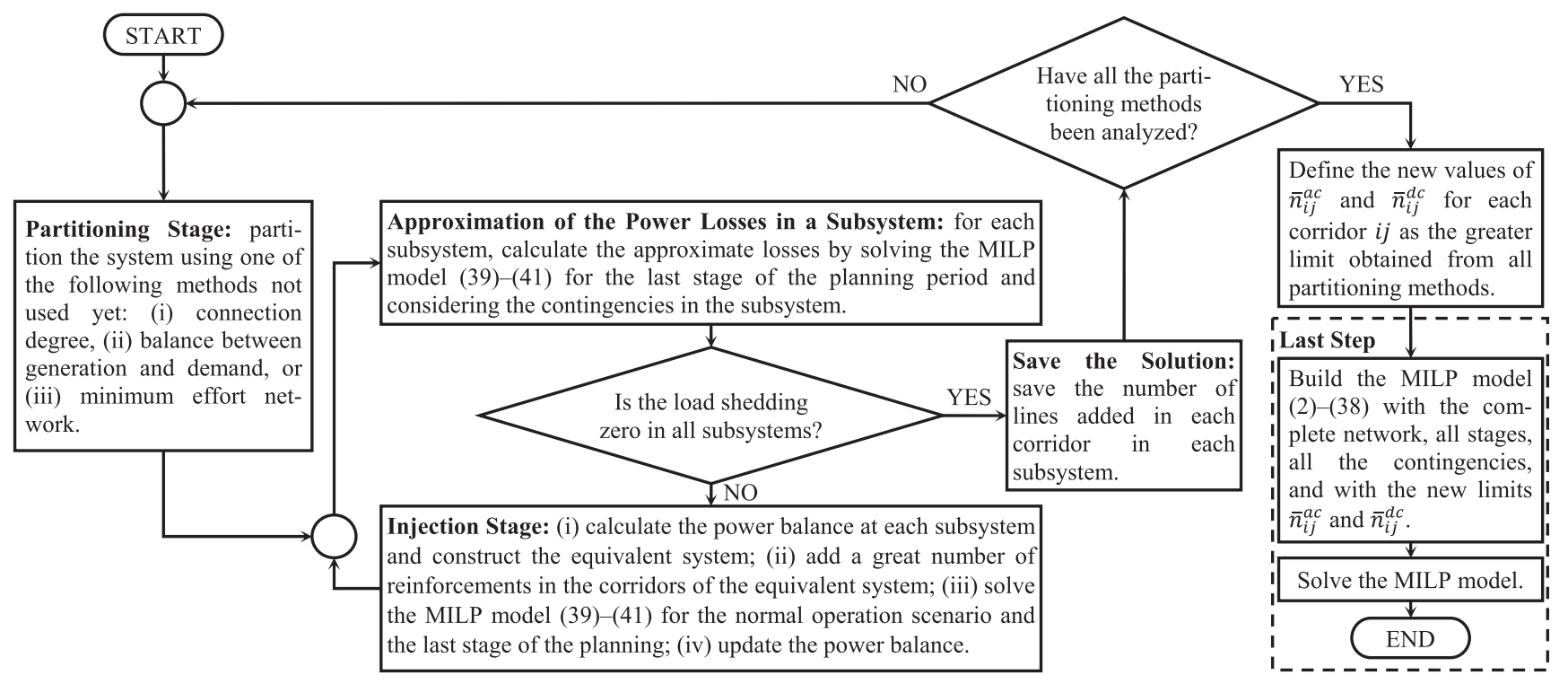

Fig. 4. Flowchart of the SSRM to solve the proposed MILP model.

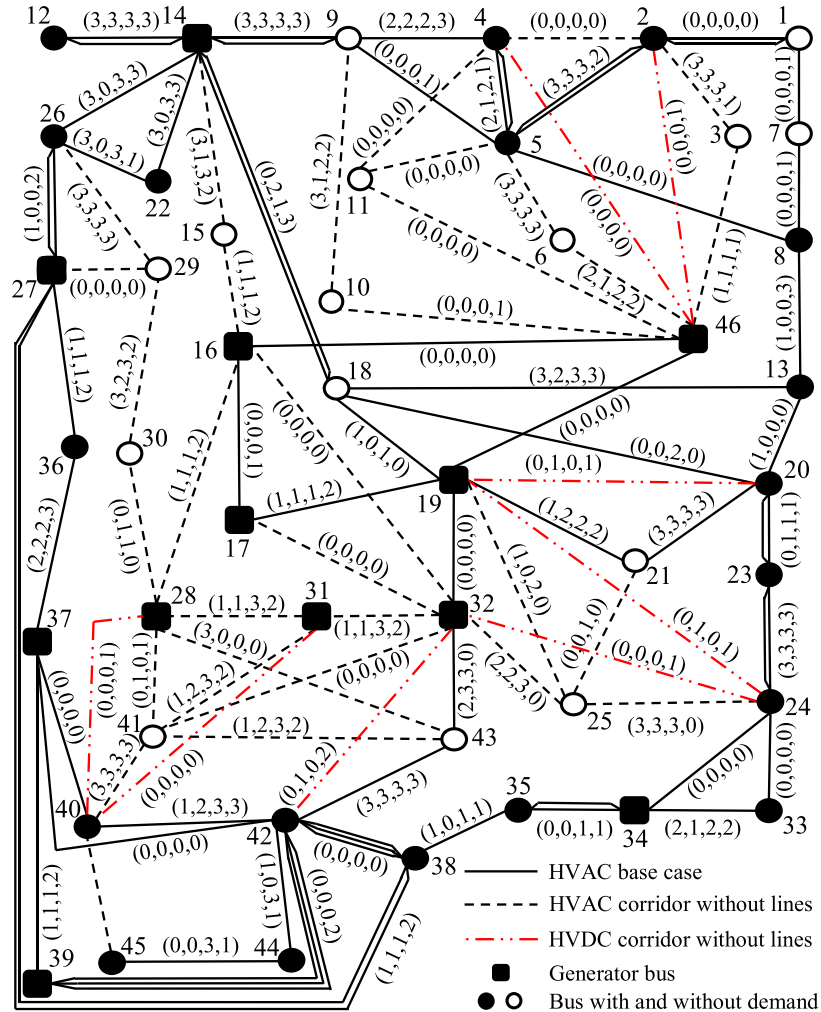

Fig. 5. Southern Brazilian system.

give evidence to the advantages of installing HVDC lines. The tests are performed with the $\mathrm{N}-1$ contingency criterion (normal operation, fifteen scenarios for contingencies in HVAC corridors and fifteen scenarios for contingencies in HVDC corridors, with a total of 31 scenarios), and the power losses are either not considered (Case A1) or considered (Case A2). For each case, four subcases are shown, with and without the candidate HVDC lines, and using and not using the SSRM. It is assumed that, in the original system, each corridor can admit a maximum of four HVAC lines and two HVDC lines. The energy cost is $C^{e}=40$ USD/MWh.

Fig. 3(e) shows the limits for the number of lines that can be added to each corridor of the system obtained with the SSRM, for cases with and without the candidate HVDC lines. The value for the corridors not listed is zero.

Table I shows the results for Garver's system. Each row of the table indicates the following: (i) rows 2-4: what is considered in each subcase; (ii) rows 5-7: the solution at each stage of planning; (iii) rows 8-11: the time needed to find the solution and the number of continuous variables, binary variables, and constraints in the problem; and (iv) rows 12-13: the planning and losses costs for the solution, shown in rows 5-7.

In Case A1, which does not consider losses, the solution is the same for all four subcases, with an investment cost of 124.18 MUSD. Even when the HVDC lines are considered, they are not added to the system, due to economic reasons. When the SSRM is used, the time needed to solve the problem is reduced from $8.25 \mathrm{~s}$ to $2.36 \mathrm{~s}$ ( $71.39 \%$ ) when HVDC lines are not considered, and from $38.97 \mathrm{~s}$ to $2.14 \mathrm{~s}(94.51 \%)$ when the HVDC lines are considered. Although losses are not considered within the optimization process in Case A1, they are approximately calculated using the solution obtained by the method.

For Case A2, which considers power losses, a different solution is found when the HVDC lines are considered. Note that the solution for Stage 1 is the same for all cases. When the HVDC lines are considered in Case A2, one bipolar line is constructed in corridor 2-6 at Stage 3. Comparing the two solutions for Case A2, by considering HVDC lines, the investment cost is reduced from 142.81 MUSD to 140.72 MUSD, and the losses cost is reduced from 17.02 MUSD to 16.98 MUSD. The same solutions are found in Case A2, with and without the SSRM. This indicates that the method reduces the search space of the problem without 
TABLE I

Garver's 6-Bus System: PlanNing Results With N-1 CRITERION

\begin{tabular}{|c|c|c|c|c|c|c|c|c|}
\hline Solution/Case & \multicolumn{4}{|c|}{ Case A1 } & \multicolumn{4}{|c|}{ Case A2 } \\
\hline Losses & \multicolumn{4}{|c|}{ No } & \multicolumn{4}{|c|}{ Yes } \\
\hline HVDC & \multicolumn{2}{|c|}{ No } & \multicolumn{2}{|c|}{ Yes } & \multicolumn{2}{|c|}{ No } & \multicolumn{2}{|c|}{ Yes } \\
\hline SSRM & No & Yes & No & Yes & No & Yes & No & Yes \\
\hline Stage 1 & \multicolumn{8}{|c|}{$(2-3),(3-5),(4-6)$} \\
\hline Stage 2 & \multicolumn{4}{|c|}{$(2-6),(3-5)$} & \multicolumn{2}{|c|}{$(2-6),(3-5),(4-6)$} & \multicolumn{2}{|c|}{$(3-5),(4-6) \times 2$} \\
\hline Stage 3 & \multicolumn{4}{|c|}{$(4-6) \times 2$} & \multicolumn{2}{|c|}{$(2-6),(4-6)$} & \multicolumn{2}{|c|}{$(2-6) \times$ 1-dc-(bipolar) } \\
\hline Time (s) & 8.25 & 2.36 & 38.97 & 2.14 & 137.56 & 27.11 & 822.73 & 54.16 \\
\hline \# of Cont. Variables & 3687 & 1896 & 9897 & 2832 & 44286 & 22863 & 125376 & 34419 \\
\hline \# of Binary Variables & 180 & 99 & 270 & 111 & 180 & 99 & 270 & 111 \\
\hline \# of Constraints & 12225 & 5658 & 29250 & 7409 & 22134 & 11364 & 56979 & 15719 \\
\hline Lines Cost (MUSD) & \multicolumn{4}{|c|}{124.18} & \multicolumn{2}{|c|}{142.81} & \multicolumn{2}{|c|}{140.72} \\
\hline Losses Cost (MUSD) & \multicolumn{4}{|c|}{20.92} & \multicolumn{2}{|c|}{17.02} & \multicolumn{2}{|c|}{16.98} \\
\hline
\end{tabular}

TABLE II

SOUTHERn BRAZILIAN SySTEM: PLANNING RESUlts With TRANSMISSION LOSSES

\begin{tabular}{|c|c|c|c|c|c|c|c|c|c|c|}
\hline Solution/Case & \multicolumn{4}{|c|}{ Case B1 (without contingencies) } & \multicolumn{4}{|c|}{ Case B2 (some contingencies) } & \multirow{2}{*}{\multicolumn{2}{|c|}{$\frac{\text { Case B3 }(\mathrm{N}-1)}{\text { Yes }}$}} \\
\hline HVDC & \multicolumn{2}{|c|}{ No } & \multicolumn{2}{|c|}{ Yes } & \multicolumn{2}{|c|}{ No } & \multicolumn{2}{|c|}{ Yes } & & \\
\hline SSRM & No & Yes & No & Yes & No & Yes & No & Yes & No & Yes \\
\hline Stage 1 & \multicolumn{4}{|c|}{$\begin{array}{c}(12-14),(20-21),(42-43) \times 2, \\
(6-46),(19-25),(24-25) \times 2, \\
(5-6) \times 2\end{array}$} & \multicolumn{2}{|c|}{$\begin{array}{c}(12-14),(19-21),(32-43) \\
(20-21) \times 3,(42-43) \times 3,(6-46) \\
(19-25),(21-25),(24-25) \times 3 \\
(5-6) \times 2 \\
\end{array}$} & \multicolumn{2}{|c|}{$\begin{array}{c}(12-14),(19-21),(32-43), \\
(20-21) \times 2,(42-43) \times 3,(6-46), \\
(5-6) \times 2,(19-24) \times 1-\text { dc-(bipolar) }\end{array}$} & - & $\begin{array}{c}(2-5),(12-14) \times 2,(19-21),(33-34),(42-44), \\
(44-45),(20-21) \times 2,(42-43) \times 2,(6-46) \times 2, \\
(31-32),(31-41),(41-43),(40-41),(5-6) \times 3, \\
(19-24) \times 1-\text { dc-(bipolar) }\end{array}$ \\
\hline Stage 2 & \multicolumn{4}{|c|}{$\begin{array}{c}(2-5),(20-21) \times 2 \\
(31-41),(40-41),(5-6)\end{array}$} & \multicolumn{2}{|c|}{$\begin{array}{l}(2-5),(31-41) \\
(40-41),(5-6)\end{array}$} & \multicolumn{2}{|c|}{$\begin{array}{c}(2-5),(20-21),(31-41), \\
(40-41),(5-6)\end{array}$} & - & $\begin{array}{c}(2-5),(20-21), \\
(42-43),(28-41)\end{array}$ \\
\hline Stage 3 & \multicolumn{4}{|c|}{$\begin{array}{l}(12-14),(19-21), \\
(17-19),(42-43), \\
(28-31),(41-43)\end{array}$} & \multicolumn{2}{|c|}{$\begin{array}{c}(12-14),(13-18),(18-20), \\
(33-34),(40-42) \times 3,(14-15) \times 2, \\
(28-31),(15-16),(40-41) \times 2\end{array}$} & \multicolumn{2}{|c|}{$\begin{array}{c}(12-14),(13-18),(17-19), \\
(20-23),(23-24),(40-42) \times 2, \\
(28-31),(41-43),(40-41) \times 2\end{array}$} & - & $\begin{array}{c}(4-5),(12-14),(13-18),(16-17), \\
(17-19),(14-22),(20-23),(23-24), \\
(40-42) \times 2,(3-46),(28-31),(40-41),(2-3) \\
\end{array}$ \\
\hline Time & $697.99 \mathrm{~s}$ & $26.57 \mathrm{~s}$ & $2509.17 \mathrm{~s}$ & $26.57 \mathrm{~s}$ & $494.71 \mathrm{~h}$ & $23.72 \mathrm{~h}$ & $565.77 \mathrm{~h}$ & $3.36 \mathrm{~h}$ & - & $697.14 \mathrm{~h}$ \\
\hline \# of Cont. Variables & 12753 & 6178 & 13473 & 6178 & 108777 & 61127 & 216609 & 60677 & 1116537 & 489677 \\
\hline \# of Binary Variables & 708 & 273 & 756 & 273 & 708 & 335 & 756 & 228 & 756 & 321 \\
\hline$\#$ of Constraints & 6849 & 3102 & 7145 & 3102 & 53941 & 29394 & 105325 & 28043 & 539053 & 231513 \\
\hline Lines Cost (MUSD) & \multicolumn{4}{|c|}{1373.33} & \multicolumn{2}{|c|}{2457.47} & \multicolumn{2}{|c|}{2331.49} & - & 3190.83 \\
\hline Losses Cost (MUSD) & \multicolumn{4}{|c|}{623.29} & \multicolumn{2}{|c|}{523.10} & \multicolumn{2}{|c|}{486.24} & - & 409.46 \\
\hline
\end{tabular}

removing the optimal solution. In this case, when the SSRM is used, the time to solve the problem is reduced from $137.56 \mathrm{~s}$ to $27.11 \mathrm{~s}(80.29 \%)$ when the HVDC lines are not considered and from 822.73 s to $54.16 \mathrm{~s}(93.42 \%)$ when the HVDC lines are considered. The reduction of the dimension of the problem achieved by the SSRM can be seen in Table I in terms of the number of variables and constraints in the problem for each subcase. For the subcases considering and not considering the HVDC lines of Case A2, the SSRM reduced the number of continuous variables in the problem by $48.37 \%$ and $72.55 \%$, binary variables by $45.00 \%$ and $58.89 \%$, and constraints by $48.66 \%$ and $72.41 \%$, respectively.

By analyzing Table I, it can be observed that both solutions for Case A2 present values for losses costs that are lower than the solution for Case A1, since the losses are considered within the optimization process for Case A2. Finally, it should be emphasized that the solution obtained for Case A1 is infeasible when losses are considered in the problem, i.e., the power capacity limit of some lines is violated in some scenarios.

\section{B. Southern Brazilian System}

The Southern Brazilian system is shown in Fig. 5. It is comprised of 46 buses and 79 corridors. The original system was modified to obtain proposals consistent with reality; the complete data for the system is available in [20]. An HVDC proposal in corridor $i j$ is considered equivalent to the same HVAC proposal in corridor $i j$, i.e., they have the same transmission capacity. The cost of building an HVAC or an HVDC line can be approximately estimated by its length, cost per mile, and some cost multipliers [21]. The cost of both the inverter and rectifier depends on their voltage level and capacity [15], which is added to the cost of the line. It is assumed that each HVAC corridor can admit a maximum of three lines and each HVDC corridor can admit a maximum of two HVDC lines. HVDC monopolar systems are proposed in corridors with lines with a capacity of less than or equal to $1000 \mathrm{MW}$, and bipolar systems are proposed in corridors with lines with a capacity greater than $1000 \mathrm{MW}$. The energy cost is $C^{e}=75 \mathrm{USD} / \mathrm{MWh}$. 
TABLE III

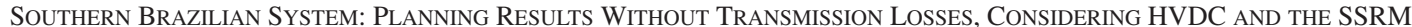

\begin{tabular}{|c|c|c|c|}
\hline Solution/Case & Case B4 (without contingencies) & Case B5 (some contingencies) & Case B6 (N-1) \\
\hline Stage 1 & $\begin{aligned}(12-14),(20-21) \times 2,(42-43) & \times 2,(6-46), \\
(25-32),(24-25),(5-6) & \times 2\end{aligned}$ & $\begin{array}{c}(12-14),(19-21),(32-43),(20-21) \times 2 \\
(42-43) \times 3,(6-46),(5-6) \times 2 \\
(19-24) \times \text { 1-dc-(bipolar) }\end{array}$ & $\begin{array}{c}(2-5),(12-14) \times 2,(19-21),(33-34),(42-44), \\
(44-45),(20-21) \times 2,(42-43) \times 2,(6-46) \times 2, \\
(31-32),(31-41),(41-43),(40-41),(5-6) \times 3, \\
(19-24) \times \text { 1-dc-(bipolar) }\end{array}$ \\
\hline Stage 2 & $(2-5),(32-43),(42-43),(31-32),(28-31),(24-25)$ & $(2-5),(20-21),(31-41),(40-41)$ & $(2-5),(20-21),(42-43),(28-41)$ \\
\hline Stage 3 & $(19-21),(20-21)$ & $\begin{array}{c}(20-23),(23-24),(40-42),(28-31),(41-43) \\
(40-41) \times 2,(5-6)\end{array}$ & $\begin{array}{c}(4-5),(17-19),(20-23),(23-24),(40-42) \times 2, \\
(3-46),(28-31),(40-41),(2-3)\end{array}$ \\
\hline Time & $5.83 \mathrm{~s}$ & $126.41 \mathrm{~s}$ & $23.02 \mathrm{~h}$ \\
\hline \# of Cont. Variables & 786 & 4611 & 40998 \\
\hline \# of Binary Variables & 273 & 228 & 321 \\
\hline \# of Constraints & 1594 & 10676 & 103047 \\
\hline Lines Cost (MUSD) & 1294.63 & 2192.85 & 3061.06 \\
\hline Losses Cost (MUSD) & 684.14 & 554.26 & 559.62 \\
\hline
\end{tabular}

The following cases, taking losses into account, are considered: (i) without contingencies (Case B1); (ii) with seventeen contingency scenarios (Case B2), normal operation and outage of lines in the HVAC corridors 19-21, 32-43, 19-25, 21-25, 25$32,20-21,24-25$, and 42-43, and outage of lines in all HVDC corridors (2-46, 4-46, 19-20, 19-24, 24-32, 32-42, 28-40, and 31-40); and (iii) N-1 security criterion (Case B3). For Cases B1 and B2, four subcases are considered, with and without candidate HVDC lines, and using and not using the SSRM. For Case B3, two subcases are considered, with candidate HVDC lines, and using and not using the SSRM.

Fig. 5 presents the solution for the limits of line additions $\left(\bar{n}_{i j}^{a c}, \bar{n}_{i j}^{d c}\right)$ of the SSRM. In this figure, for example, if the HVAC corridor 40-42 is considered, the limits $(1,2,3,3)$ are used to obtain each expansion solution presented in Table II; i.e., Case B1 uses the limit $\bar{n}_{40-42}^{a c}=1$ for the two subcases when the SSRM is used, Case B2 uses the limit $\bar{n}_{40-42}^{a c}=2$ for the tests when the HVDC lines are candidates and the limit $\bar{n}_{40-42}^{a c}=3$ for the test when the HVDC lines are not candidates and the SSRM is used, and Case B3 uses the limit $\bar{n}_{40-42}^{a c}=3$ when the SSRM is used. In other words, each position of the limits solution corresponds to the limit used in each case to find the planning solutions in Table II.

The results for Cases B1-B3 are shown in Table II. For Case B1, the solution is the same for all the subcases, with an investment cost of 1373.33 MUSD and losses cost of 623.29 MUSD, indicating that even if HVDC lines are considered, they are not installed, because they are not an attractive alternative for the situation without contingency scenarios. The use of the SSRM reduces the computational times by $96.19 \%$ and $98.90 \%$ for the cases not considering and considering the HVDC lines, respectively, without excluding the optimal solution of the problem.

For Case B2, by considering the candidate HVDC lines, the investment cost is reduced by $5.13 \%$, and the losses cost is reduced by $7.05 \%$. The use of the SSRM reduces the computational time by $95.21 \%$ when the candidate HVDC lines are not considered and by $99.41 \%$ when the candidate HVDC lines are considered in the planning process, again, without excluding the optimal solution of the problem. This gives clear evidence to the method's potential for identifying relevant corridors in the system to reduce the computational effort in the TNEP problem.

For Case B3, a solution could only be obtained using the SSRM. Because of the size of the problem, the computational time to solve the problem is $697.14 \mathrm{~h}$, and the cost of investment increased by $36.86 \%$ in relation to Case B2 (considering the candidate HVDC lines), where only the contingencies in the main corridors are considered. The cost of losses of the solution in Case B3 is smaller than that in Case B2, because more lines are constructed, which implies a lower value of losses.

Three other cases are tested without losses, considering the HVDC lines, and using the SSRM: (i) Case B4, without contingencies; (ii) Case B5, with the same contingencies as Case B2; and (iii) Case B6, with $\mathrm{N}-1$ security criterion. The results for cases B4-B6 are shown in Table III. The limits of the line additions used in these cases are the same as those used for Cases B1-B3 when the HVDC lines were considered.

By comparing Cases B4 and B1, B5 and B2, and B6 and B3 (when $\mathrm{B} 1, \mathrm{~B} 2$, and $\mathrm{B} 3$ are considering the candidate HVDC lines and using the SSRM), it can be seen that the solution for the problem, without considering losses, presents a lower investment value. This is expected, first, because losses are not optimized in these three last cases and second, because losses consideration implies larger capacities for the corridors in the system, which can be accomplished by installing more lines. Although the losses are not optimized in the cases shown in Table III, they are calculated using the solution obtained. The cost of losses in Cases B4-B6 are higher than the ones in Cases B1-B3, since they are not considered within the optimization process, as in Case A1 of Garver's system. Note that when the solutions for Cases B3 and B6 are compared, the total value, i.e., lines cost plus losses cost, is greater for Case B6 (3620.68 MUSD) than for Case B3 (3600.29 MUSD), in which losses are included. This shows the importance of including losses in the optimization method.

Finally, by comparing the times needed to find the solutions in Table II and Table III, a high increase in the computational effort can be verified when the power losses are included in 
the model. For example, comparing Cases B3 (considering the candidate HVDC lines) and B6, the computational time is 29.28 times higher when losses are taken into account in the problem. For Case B3, the SSRM reduced the number of continuous variables in the problem by $56.14 \%$, binary variables by $57.54 \%$, and constraints by $57.05 \%$. For that reason, it is important to develop methods to reduce the search space of the problem without excluding excellent solutions.

\section{CONCLUSION}

This work presented a method to solve the multistage security-constrained transmission expansion planning problem using a mixed-integer linear programming model that included HVAC and HVDC proposals and power losses, and considered a reduced search space. The objective was to minimize the total cost of planning and power losses in all stages.

The results for Garver's and the Southern Brazilian systems show that the inclusion of HVDC lines in the transmission planning helps to improve the performance of the network and reduce the cost of investment in the future, thereby improving the expansion plan. The results also show that there exists a tendency to install bipolar HVDC lines to improve the reliability of the system. Also, the inclusion of the losses in the analysis reflects a tendency to add more lines in the expansion plan, and can lead to better solutions when the overall cost of investments and operation is considered.

For the tests performed, the search space reduction strategy obtained new limits for line additions in each corridor of the system, thereby reducing the computational effort to solve the problem considerably, without excluding its optimal solution.

\section{REFERENCES}

[1] G. Latorre, R. D. Cruz, J. M. Areiza, and A. Villegas, "Classification of publications and models on transmission expansion planning," IEEE Trans. Power Syst., vol. 18, no. 2, pp. 938-946, May 2003.

[2] H. Zhang, V. Vittal, G. Heydt, and J. Quintero, "A mixed-integer linear programming approach for multi-stage security-constrained transmission expansion planning," IEEE Trans. Power Syst., vol. 27, no. 2, pp. 1125-1133, May 2012.

[3] J. D. Molina and H. Rudnick, "Transmission of electric energy: A bibliographic review," IEEE Latin Amer. Trans., vol. 8, no. 3, pp. 245-258, Jun. 2010.

[4] S. Lumbreras and A. Ramos, "The new challenges to transmission expansion planning. Survey of recent practice and literature review," Elect. Power Syst. Res., vol. 134, pp. 19-29, May 2016.

[5] C.-K. Kim, V. K. Sood, G.-S. Jang, S.-J. Lim, and S.-J. Lee, HVDC Transmission: Power Conversion Applications in Power Systems. Singapore: Wiley, 2009.

[6] M. Henderson, J. Gagnon, D. Bertagnolli, B. Hosie, G. DeShazo, and B. Silverstein, "Building a plan for HVDC," IEEE Power Energy Mag., vol. 5, no. 2, pp. 52-60, Mar./Apr. 2007.

[7] M. P. Bahrman and B. K. Johnson, "The ABCs of HVDC transmission technologies," IEEE Power Energy Mag., vol. 5, no. 2, pp. 32-44, Mar./Apr. 2007.

[8] "ABB review special report: 60 years of HVDC," ABB Technol. Ltd., Zurich, Switzerland, ABB Rev. Tech. Rep. 2014. [Online]. Available: https://library.e.abb.com/public/fcf44b87ad51488d87b3de433abb7273/ HVDC\%20special\%20report\%20-\%20FINAL_2.pdf, Accessed on: Feb $5,2016$.

[9] C. Liu, B. Zhang, Y. Hou, F. F. Wu, and Y. Liu, "An improved approach for AC-DC power flow calculation with multi-infeed DC systems," IEEE Trans. Power Syst., vol. 26, no. 2, pp. 862-869, May 2011.

[10] R. L. Hauth, P. J. Tatro, B. D. Railing, B. K. Johnson, J. R. Stewart, and J. L. Fink, "HVDC power transmission technology assessment," Oak
Ridge Nat. Lab., Oak Ridge, TN, USA, Tech. Rep. ORNL/Sub/95SR893/1, Apr. 1997.

[11] M. L. Gilles, "Optimum HVDC-transmission expansion planning - A new formulation," IEEE Trans. Power Syst., vol. 2, no. 2, pp. 429-435, May 1987.

[12] A. Lotfjou, Y. Fu, and M. Shahidehpour, "Hybrid AC/DC transmission expansion planning," IEEE Trans. Power Del., vol. 27, no. 3 pp. 1620-1628, Jul. 2012.

[13] E. F. Silva, M. Rahmani, and M. J. Rider, "A search space reduction strategy and a mathematical model for multi-stage transmission expansion planning with N-1 security constrains," J. Control, Autom. Elect. Syst., vol. 26, no. 1, pp. 57-67, Feb. 2015.

[14] M. Rahmani, R. Romero, and M. J. Rider, "Strategies to reduce the number of variables and the combinatorial search space of the multi-stage transmission expansion planning problem," IEEE Trans. Power Syst., vol. 28, no. 3, pp. 2164-2173, Aug. 2013.

[15] A. J. Jardini and J. F. Nolasco, "Impacts of HVDC lines on the economics of HVDC projects," CIGRÉ-Working Group B2/B4/C1.17, Paris, France, Tech. Brochure 388, Aug. 2009. [Online]. Available: http:// b4.cigre.org/Publications/Technical-Brochures/TB-388-2009-JWG-B2B4-C1.17-IMPACTS-OF-HVDC-LINES-ON-THE-ECONOMICS-OFHVDC-PROJECTS, Accessed on: Oct. 5, 2015.

[16] S. A. Ross, R. W. Westerfield, and J. Jaffe, Corporate Finance, 9th ed. New York, NY, USA: McGraw-Hill/Irwin, 2010.

[17] R. Billinton, M. Fotuhi-Firuzabad, S. O. Faried, and S. Aboreshaid, "Composite system reliability evaluation incorporating an HVDC link and a static synchronous series compensator," in Proc. Can. Conf. Elect. Comput. Eng., May 2002, pp. 42-47.

[18] N. Alguacil, A. L. Motto, and A. J. Conejo, "Transmission expansion planning: A mixed-integer LP approach," IEEE Trans. Power Syst., vol. 18, no. 3, pp. 1070-1077, Aug. 2003.

[19] A. Duque, A. Escobar, and R. A. Gallego, "Multi-stage transmission expansion planning via network partitioning and principal variables identification," in Proc. 2014 IEEE PES Transm. Distrib. Conf. Expo.-Latin Amer. Sep. 2014, pp. 1-6.

[20] "GP Power System Test Cases Repository." [Online]. Available: http:// academia.utp.edu.co/planeamiento/?p $=2521$, Accessed on: Jan. 15, 2016.

[21] M. Tim, C. Trevor, and W. Dan, "Capital costs for transmission and substations: Recommendations for WECC transmission expansion planning," Oct. 2012. [Online]. Available: https://www. wecc.biz/Reliability/1210_BV_WECC_TransCostReport_Final.pdf, Accessed on: Jan. 10, 2016

Andres Hernando Dominguez received the B.Sc. and M.Sc. degrees in electrical engineering from the Technological University of Pereira, Pereira, Colombia, in 2009 and 2012, respectively, where he is currently working toward the Ph.D. degree in electrical engineering. His areas of research are the development of methods for the optimization and planning of electrical power systems.

Leonardo H. Macedo (S'14) received the B.Sc. and M.Sc. degrees in electrical engineering, in 2012 and 2015, respectively, from the São Paulo State University, Ilha Solteira, Brazil, where he is currently working toward the Ph.D. degree in electrical engineering. His current research interests include the development of methods for the optimization, planning, and control of electrical power systems.

Antonio Hernando Escobar received the B.Sc. degree in electrical engineering in 1987 and the M.Sc. degree in 2002, both from the Technological University of Pereira, Pereira, Colombia, and the Ph.D. degree from the São Paulo State University, Ilha Solteira, Brazil, in 2009. He is currently a Professor of electrical technology at the Technological University of Pereira, Pereira, Colombia. His general research interest is in the field of electrical power systems planning.

Rubén Romero (M'93-SM'08) received the B.Sc. and P.E. degrees from the National University of Engineering, Lima, Perú, in 1978 and 1984, respectively. $\mathrm{He}$ received the M.Sc. and Ph.D. degrees from the University of Campinas, Campinas, Brazil, in 1990 and 1993, respectively.

He is currently a Professor of electrical engineering at the São Paulo State University, Ilha Solteira, Brazil. His research interests include methods for the optimization, planning, and control of electrical power systems, applications of artificial intelligence in power systems, and operations research. 